\title{
XIV.
}

\section{Ueber 0esophagitis dissecans superficialis mit einem Beitrag zur Kenntniss des Epithels des Oesophagus des Menschen.}

\author{
Von Dr. Georgios Sclavunos, \\ I. Assistenten am anatomischen Institut zu. Würzburg.
}

(Hierzu Taf. VI.)

Unter obiger Bezeichnung wurde neuerdings von Rosenberg ${ }^{1}$ ) eine Erkrankung der Oesophagusschleimhaut beschrieben, wobei sich die Epitheldecke röhrenförmig ablöst und ausgestossen wird. Aehnliche Fälle unter anderer Benennung sind von BirchHirschfeld ${ }^{2}$ ) und Reichmann ${ }^{3}$ ) veröffentlicht worden. Im Folgenden werde ich einen vierten Fall auseinandersetzen, der wegen seiner Aetiologie und pathologischen Veränderungen zur Erkenntniss dieser seltenen Erkrankung der Speiseröhre beitragen dürfte. Vorher möchte ich kurz auf die drei übrigen bis jetzt bekannten Fälle eingehen.

Birch-Hirschfeld erwähnt in seinem Lehrbuch einen von Dr. Nieder in Dresden beobachteten Fall, welcher eine hysterische Dame betraf. Dieselbe bekam plötzlich Schmerzen am Halse, Unfähigkeit zu Schlucken, Temperaturerhöhung und erbrach am dritten Tage eine etwa $20 \mathrm{~cm}$ lange, häutige Röhre von grauem, etwas durchscheinendem Aussehen. Nach 8 Tagen genas die Patientin völlig. Die mikroskopische Untersuchung bewies, dass die Membran die in toto ausgestossene Epitheldecke des Oesophagus war; ihre oberste Schicht war von normalem Aussehen, hingegen waren die untersten Zellschichten auf das

1) Oesophagitis dissecans superficialis. Centralblatt für allgemeine Pathologie u. patholog. Anatomie. No. 18. 1892.

2) Birch-Hirschfeld, Lebrbuch der patholog. Anatomie. 3. Auflage. Bd. II. S. 511.

3) Deutsche med. Wochenschr. 1890. No. 46. 

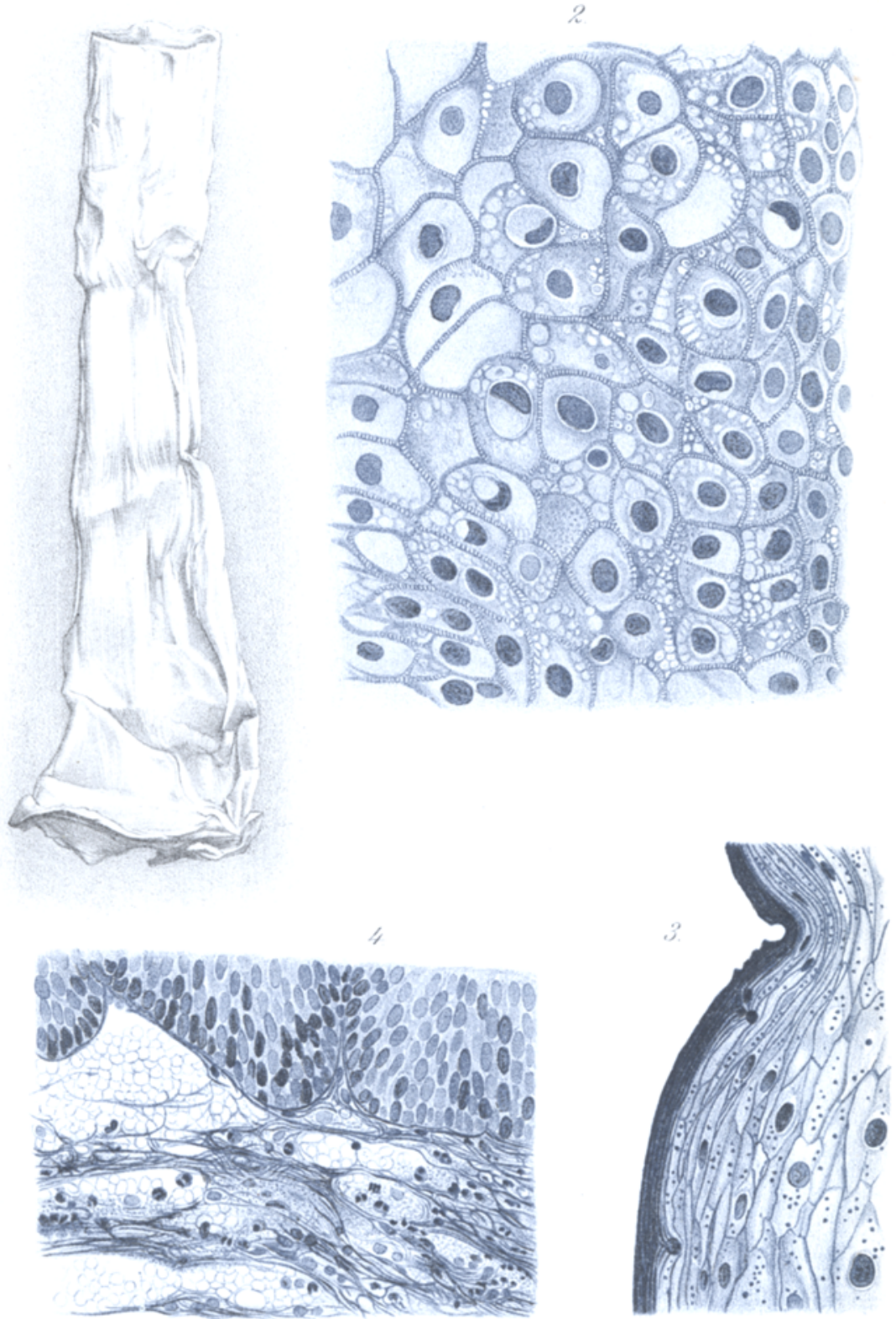
dichteste von Rundzellen durchsetzt. Birch-Hirschfeld bezeichnet die Erkrankung als eine pseudocroupöse Entzündung, indem er mit grösster Wahrscheinlichkeit in der subepithelialen Entzündung die Veranlassung zur Ablösung der Epitheldecke annimmt.

Im Gegensatz zu diesem Falle sollen bei dem von Reichmann veröffentlichten langjährige Schluckbeschwerden vorangegangen sein. Eines Tages blieb dem Patienten ein Bissen Fleisch im Oesophagus stecken, der ihm das Verschlucken unmöglich machte. Nach 5 Tagen entleerte der Patient unter Brechbewegungen eine ziemlich grosse Membran. Letztere wurde Reichmann in Fetzen von verschiedener Grösse übergeben, die zusammen eine Fläche von $100 \mathrm{ccm}$ ausmachten. Einige Stäcke der erbrochenen Membran waren $15 \mathrm{~cm}$ lang. Die Dicke überstieg nicht $\frac{1}{8} \mathrm{~mm}$. Die mikroskopische Untersuchung ergab, dass die Membran aus mehreren Schichten von Pflasterepithelzellen mit grossem Kerne bestand. Die Zellen unterschieden sich nicht von normalen, und zwischen ihnen waren Spalten za sehen. Aus ihrem Verhalten gegen Alkalien, sowie gegen Hämatoxylin und Pikrocarmin schliesst Reichmann auf Verhornung derselben und nimmt zum Schluss an, dass bei dieser Erkrankung der Oesophagusschleimhaut nebst der Hyperproduction von Epithelzellen eine Kittsubstanz sich bildet, welche die Zellen zusammenhält und so die Bildung einer Membran ermöglicht. Von einer Infiltration der untersten Epithelzellen mit Rundzellen wird nichts erwähnt, auch vermisst man in diesem Falle genaue Angabe, ob es sich um die Ablösung der ganzen Epitheldecke oder der oberflächlichen Schicht derselben handelt. Reichmann schlägt den Namen Oesophagitis exfoliativa vor.

Der letzte bis jetzt bekannte und von Rosenberg veröffentlichte Fall betrifft einen jungen Mann von 20 Jahren, der seit 2 Jahren an Dyspepsie litt und bei welohem es ohne irgend eine vorhergegangene Störung seines Befindens unter starken Brechbewegungen, begleitet von einem Erstickungsgefühl, zur Entleerung einer röhrenförmigen Membran kam. Dieselbe war stellenweise mit blutigen Streifen befleckt und hatte eine Länge von $18 \mathrm{~cm}$ und eine Breite von ungefähr $2 \frac{1}{2} \mathrm{~cm}$. Ihre äussere Fläche war in Falten geschrumpft, ziemlich glatt, dagegen zeigt 
die innere Oberfläche ein mattes Aussehen, als ob sie mit Kleie bestäubt wäre. Ob die innere Fläche der erbrochenen Röhre der ursprünglichen, normalerweise dem Lumen des Oesophagus zugewandten entsprach, oder ob eine Umstülpung der entleerten Röhre stattgefunden hatte, darüber bemerkt Rosenberg nichts. Er findet ferner eine Erweiterung des Oesophagus, da das cardiale Ende der entleerten Röhre $25 \mathrm{~mm}$ Breite hatte. Was die Dicke betrifft, so war sie nicht überall dieselbe; sie variirte zwischen 1-3 $\mathrm{mm}$ und war, verglichen mit der vom Fall Reichmann's, 25mal stärker. Bei der mikroskopischen Untersuchung liessen sich folgende fünf "histoide Strukturbestandtheile" feststellen. 1) als Hauptelement erschienen normale, grosse und lebenskräftige Pflasterepithelzellen mit färbbarem, ovalem Kern. 2) eine dünne fibrilläre Masse mit einer Körnung zwischen den Fibrillen und mit darin eingebetteten Leukocyten. Diese Masse lag an der unteren Fläche der Epitheldecke, die an die Tunica propria grenzt. 3) Massen, welche aus schüppchenartigen, concentrisch geschichteten Epithelzellen ohne Kern bestanden, die Farbe schlecht annahmen und welche an die bei Cancroid vorkommenden Perlkugeln erinnerten mit umgekehrter Anordnung der "succulenten" Zellen, indem letztere hier die Mitto der Masse einnahmen. 4) kugelförmige Conglomerate, die "aus angehäuften und gleichsam zusammengeklebten Leukocyten bestanden " und die an der Papillarfläche des Epithels sassen. 5) kugelförmige Körper, die sich aus "succulenten" Epithelzellen zusammensetzten und in der sub 1) erwähnten fibrillär-körnigen Masse ihre Lage hatten.

Rosenberg hält die letztangeführten, kugelförmigen Körper nicht fär carcinomatöse Gebilde, an die man gedacht hätte, da sie nicht im Bindegewebe lagen, und erinnert daran, dass J. Klein solche Perlkugeln in der Nachbarschaft um einen abgelaufenen Prozess beobachtet hat, in Geweben, die mit einem Epithellager ausgestattet waren. Was die Aetiologie und das Zustandekommen der röhrenförmigen Ablösung des Epithelüberzugs des Oesophagus betrifft, so erfährt man auch aus diesem Falle nichts Weiteres.

Von unserem Falle ist mir anamnestisch durch die Güte von Dr. Grätzer, aus dessen Praxis er stammt, Folgendes mitgetheilt worden. 
Der Zuchthäusler A. G., 25 Jahre alt, hat wiederholt „ganz starken Spiritus getrunken, den er zu mechanischer Verwendung gehabt bat". Am 27. Angust bekam er plötzlich Blutbrechen, wobei er angeblich gegen 2 Liter Blut verioren haben soll. Bei der Untersuchung des Rachens fand der Arzt zum Munde heraus eine röhrenförmige Membran von etwa $15 \mathrm{~cm}$ Länge hängen. Beim Versuchen dieselbe herauszuziehen, klagte Patient über starke Schmerzen, so dass der Arzt mit der Scheere den grössten Theil derselben abschneiden musste, während der übrige Theil in den Oesophagus zurückglitt. Eispillen und gekühlte Nilch wurden 5 Tage hindurch verabreicht, wonach vollständige Genesung obne Narbenbildung erfolgte. Alles Essen wurde vertragen. Patient ist vollständig wieder gesund. Da der Arzt nicht sicher war, um was für ein Gewebe es sich handelt und da beim Patienten Simulation in Betracht kam, schickte er mir den ausgestossenen Gewebstheil in Spiritus eingelegt.

Derselbe zeigt sich makroskopisch als eine membranöse Röhre von etwa $10 \mathrm{~cm}$ Länge (Fig. 1). Das eine Ende derselben ist breiter und hat einen unregelmässigen Rand, wäbrend das andere einen scharfen Rand zeigt. Welches von beiden Enden das cardiale ist, lässt sich ohne Weiteres nicht sagen. Wie wir jedoch weiter unten sehen werden, entspricht das erweiterte Ende der Gegend der Cardia. Was die Dicke der Röbrenwand betrifft, so ist sie nicht überall dieselbe; am dünnsten ist sie an dem erweiterten Ende. Die äussere Fläche ist, abgesehen von den Falten, die durch die Behandlung hier stärker hervortreten, ziemlich glatt, die innere Fläcbe zeigt ein weissliches, mattes Aussehen, und liegen derselben weisse, fibrinartige Fäden an.

Zur Beschreibung der mikroskopischen Befunde übergebend, bemerke ich zugleich, dass ich zur sicheren Beurtheilung der.hierbei auffallenden Veränderungen mikroskopische Präparate aus 2 frisch der Leiche entnommenen Speiseröhren angefertigt und zur Vergleichung herangezogen habe. Um dem Einwand vorzubeugen, dass die gleich zu besprechenden Veränderungen der Behandlung der ausgestossenen Röhre ihre Entstehung verdanken, brachte ich die normalen Speiseröhren behufs Härtung direct in Spiritus. -

Zur mikroskopischen Untersuchung der entleerten Membran wurden Stückchen aus dem oberen, mittleren und unteren Theil verwendet. Als Farbe kam Delafield's Hämatoxylin hauptsächlich in Anwendung. - Betrachtet man einen Schnitt, der durch die ganze Wand der Röbre geführt worden ist, bei schwacher Vergrösserung, so fällt zuerst auf, dass gegen das Lumen der Röhre nicht die oberflächliche Schicht der Epitheldecke, sondern die Keimschicht derselben mit ihren Papilleneindrücken zugewendet ist. Es muss also bei der Ablösung und Ausstossung der Röhre eine Umstülpung derselben vor sich gegangen sein, so dass die sonst normaler Weise dem Lumen des Oesophagus zugekehrte Oberfläche der Oesophagusschleimhaut an der entleerten Röhre nach aussen zu liegen kam. Man constatirt ferner bei dieser Vergrösserung, dass die ganze Wand der Röbre allein aus dem vollständigen, scheinbar unversehrt erhaltenen Epithelstratum der Oesopbagusschleimhaut besteht. Nur an Schnitten aus dem mittleren und bie und da 
auch aus dem pharyngealen Theil findet man noch anderes Gewebe der Keimschicht des Epithels anliegend, welches offenbar den weiter oben erwähnten makroskopisch sichtbaren, fibrinartigen Auflagerungen entspricht. Das Epithel selbst besteht (bei schwacher Vergrösserung) in der mittleren Schicht aus lauter hellen Zellen, die wenig Farbe oder gar nicht angenommen baben. Die Dicke des Epithels ist verschieden; an einigen Schnitten war es sehr dünn und wie atrophisch. Die oberste Schicht des Epithels zeigt auch eine verschiedene Dicke, an einigen Stellen sah man zapfenförmige Verdickungen, die in die mittlere Schicht des Epithels eingesenkt waren.

Bei Anwendung von starker Vergrösserung lassen sich folgende Veränderungen beobachten. Sie bestehen erstens daria, dass die untersten und mittleren Zellen bis zur oberfächlichsten Schicht des Epithels einen aufgequollenen Kern zeigen, dessen Substanz vermindert ist und dessen Membran selbst hie und da aufgelöst war. Zweitens sieht das Zellprotoplasma fast homogen aus, der Zellleib aufgequollen, und was mehr auffällt, man erkennt darin runde Hohlräume, die öfters zu mehreren neben einander liegen (Fig. 2). Das ganze Aussehen der Epithelzellen erinnert sehr an das der bydropischen Degeneration derselben. Mit diesen Hoblräumen, die ihren Sitz im Zellinnern hatten, waren leicht zu verwechseln runde Hohlräume, die von feinen protoplasmatischen Fäden umgrenzt waren, welche von einer Zelle zur anderen zogen.

Wie man aus der normalen Histologie durch die Untersuchungen von Bizzozzero'), Ranvier ${ }^{2}$ ), Flemming ${ }^{3}$ ), Retzius ${ }^{4}$ ) u. A. weiss, sind die Zellen der Malpighischen Schichte der Epidermis durch protoplasmatische Brücken, welche im Innern der Zelle mit einem Netz zusammenhängen, verbunden; zwischen denselben befinden sich die Intercellularräume, die mit den Lymphräumen der Cutis, wie es Retzius durch Injectionen bewies, zusammenhängen. Klein wies dasselbe Verhältniss für die Epithelzellen der Schleimhäute, von dem gegenseitigen Verhalten der Epithelzellen des Oesophagus jedoch ist mir wenigstens nichts bekannt.

1). Sulla Struttura degli Epitheli parimentos stratificati. Centralblatt. 1871. Ueber den Bau der geschichteten Plattenepithelien in: Moleschott's Untersuchungen. Bd. II. 1872.

2) Sur la structure des cellules du corps muqueuse de Malpighi. Comptes rendus. T. 95 . p. 1374.

3) Zellṣubstanz, Kern und Zelltheilung. S. 53 .

4) Zur Kenntniss der Saftbahnen in der Haut des Menschen. Biologische Untersuchungen. 1881. 
Die oben erwäbnten, zwischen den Zellen liegenden, von den feinen Fäden umgrenzten Hoblräume entsprechen unzweifelbaft den abnorm erweiterten und gedehnten Intercellularlücken and Intercellularbrücken. In den Intercellularlücken konnte man öfters Leukocyten wahrnehmen; eine Leukocyteninfiltration des Epithels in abnormer Weise war jedoch nicht zu constatiren.

Die oberste Epithelschicht unterschied sich nicht von jener eines normalen Prăparates, nur an den oben erwähnten verdickten Stellen waren die Zellen stärker zusammengeschrumpft. Sie bestand aus abgeplatteten Zellen mit einem meistens stäbchenförmigen Kerne. Unter dieser obersten Epithelschicht lag eine Zellreibe mit einem körnigen Inhalt. Die Körner waren intensiv blau gefärbt. Eine Untersuchung mit Alkalien und Acidum glaciale erwies, dass es sich um Eleidin- oder Keratohyalinkörner handelt (Fig. 3). Wie man aus den Uutersuchungen von Ranvier ${ }^{1}$ ), Waldeyer ${ }^{2}$ ) u. A. weiss, kommt diese körnige Substanz dort vor, wo die Zellen von Pflasterepithelien der Verhornung anheimfallen, und steht unzweifelbaft mit dem Verhornungsprozess in Zusamenenbang. Vom normalen Vorkommen des Eleidins im Epithel der Speiseröhre von Thieren weiss man schon ${ }^{3}$ ), ob es aber auch im Oesophagusepitbel des Menschen vorkommt, darüber liegen keine Angaben vor. Daher glaubte ich anfangs mit mehr Recht als Reichmann (a. a. 0.) auf eine Verbornung der Epithelzellen zu schliessen und mit dieser Verbornung den Erkrankungsprozess in Zusammenhang zu bringen. Die Besichtigung jedoch meiner normalen Oesophaguspräparate ergab, dass das Eleidin a uch da vorhanden ist, allerdings in nicht so reichlicher Menge wie an einigen Stellen der entleerten Membran. Am besten bringt man es durch Flächenschnitte zur Anschauung. Auch an friscben Präparaten äberzeugte ich mich von dessen Vorkommen. Somit ist den Angaben Reichmann's von verbornten Zellen des Epitbels der ausgestossenen Röbre für den Erkrankungsprozess keine Bedeutung zuzuschreiben, da eine wenn auch geringe Verhornung der Epithelzellen des Oesophagus des Menschen normaler Weise vor sich geht.

Wie weiter oben bemerkt, bestand die ganze Röhrenwand hauptsächlich auș dem Epithelüberzug der Oesophagusschleimhaut. Es hat Stellen jedoch

1) Sur une substance nouvelle de l'epiderme et sur le prozessus de keratinisation du revêtement épidermique. Compt. rend. 1879. p. 1361.

2) Untersuchungen über die Histogenese der Horngebilde, insbesondere der Haare und Federn. Festgabe Henle's. Bonn 1882. Verlag von Max Cohen und Sohn.

3) Ranvier, De l'Eleidin et de la reparation de cette substance dans le peau, la muqueuse buccale et la muqueuse oesophagienne des vertebrés. Archiv de Physiologie. T. XVI. p. 125. - Sclav u n os, Untersuchungen über das Eleidin und den Verbornungsprozess an der Pars cardiaca des Magens der Säugetbiere. Verbandlungen der physikal.-medicinischen Gesellschaft zu Würzburg. N. F. Bd. XXIV. S. 161. 
gegeben, welche Reste von subepithelialem Gewebe aufwiesen. Man konnte hierbei sehr leicht verfolgen, wie das subepitheliale Bindegewebe sich allmählich vom Epithelstratum ablöste. An den am wenigsten veränderten Stellen sass das Epithel fest auf dem fibrillären Bindegewebe der Propria, die Papillen fügten sich in die entsprechenden Eindrücke des Epithels ein. Betrachtete man andere Stellen, so beobachtete man, wie das Bindegewebe von der Keimschicht des Epithels abgedrängt war, die Papillen selbst niedergedrückt, so dass ein Raum zwischem dem Epithel und dem Bindegowebe zurückblieb, welcher von Leukocyten und von einer feinkörnigien, fädigen Substanz eingenommen wurde. Diese Substanz mit den Leukocyten lagerte sich auch zwischen den Bindegewebsfibrillen und schien dieselbe auseinander gedrängt zu haben (Fig. 4). Oefters war sie zu rundlichen Massen zusammengeballt, in welchen Leukacyten sich befanden; man konnte ausserdem in der Mitte oder an der Peripherie dieser rundlichen Massen Blut- und Lymphgefässe erkennen. Es machte daher den Eindruck, als ob es sich um eine eiweissartige Substanz (Fibrin) bandle, die besonders um die Gefässe herum zur Gerinnung kam. Ferner fanden sich unter dem Epithel kleine Blutungen, besonders an jenen Stellen, wo das Bindegewebe der Tunica propria nicht so sehr verändert war (Fig. 4). Offenbar muss an diesen. Stellen eine gewaltige Ablösung des Epithels stattgehabt haben.

Rosenberg erwähnt in seinem Falle rundlicber Körper, die aus "succulenten" Epithelzellen bestanden und die unter dem Epithel in einer fibrillären Masse eingebettet waren, wovon ich in meinem Falle nichts babe beobachten können. Ohne die Angaben von Rosenberg anzweifeln zu wollen, möchte jch darauf binweisen, dass ich auch rundliche, aus Epithelzellen bestehende Körper an einzelnen Schnitten beobachtete, es stellte sich jedoch beim Serienschneiden heraus, dass dieselben nichts anderes waren, als abgeschnittene Theile der schief vom Messer getroffenen kolbigen Auftreibungen des Epithels. War unter dem Epithel die oben genannte feinfädige Substanz vorbanden, so machte es den Eindruck, als ob diese rundlichen Massen, ohne in Zusammenhang mit dem Epithel zu stehen, für sich in dieser Substanz eingebettet lagen.

Wenn man jetzt die Veränderungen der übrigen drei Fälle, soweit dieselben genau untersucht worden sind, mit denen vorliegenden Falles in Vergleich zieht, so findet man, dass dioselben in vielen Punkten übereinstimmen. Danach scheint der Erkrankangsprozess sich im Epithel und dem subepithelialen Bindegewebe abzuspielen. Welche Ursachen demselben zu Grunde liegen mögen, ist aus den drei übrigen Fällen nicht bekannt.

Im vorliegenden Falle ist uns aus der Anamnese bekannt, dass Patient mehrmals vor der Erkrankung starken Spiritus zu sich nahm, welcher ihm im Zuchthaus zu mechanischen Zwecken 
gewährt worden war. Aus der Literatur ist mir leider nichts bekannt über die Einwirkung starken Spiritus auf Schleimhäute; trotzdem ist es mir nicht unwahrscheinlich, dass die oben beschriebenen Veränderungen ihre Ursache in der wiederholten Einwirkung des starken Spiritus haben. Und in dieser Beziehung möchte ich auf die aus der pathologischen Anatomie bekannten Veränderungen der Oesophagusschleimhaut hinweisen, die man bei Potatoren antrifft. Die Einwirkung ist im vorliegenden Falle so zu denken, dass der Spiritus zuerst das Epithel, welches bekanntlich für Flüssigkeit sebr durchlässig ist, alterirt und dann auf die subepithelialen Gefässe entzündend eingewirkt habe, so dass es zu einer Durchtränkung der Propria der Schleimhaut mit einem serösen Exsudat gekommen ist.

Dafür spricht die Aufquellung und Vacuolenbildung der Epithelzellen, sowie die feinkörnige Substanz nebst den Leukocyten. $O b$ die abnorme Erweiterung der Intercellularlücken der Einwirkung des Agens zuzuschreiben sind, das lässt sich mit Sicherheit nicht behaupten, da Mitrophanow ${ }^{1}$ ) solche abnorm erweiterte Intercellularlücken durch mechanische Reizung an der Seitenhaut von Tritonen erzeugte und, wie weiter unten ersichtlich, eine solche Reizung hat bei der Ausstossung der Epithelröhre im vorliegenden Falle unzweifelhaft stattgefunden. Die subepitheliale Entzündung bewirkte durch das Exsudat eine allmähliche Lockerung des Epithels von seiner bindegewebigen Unterlage. Die wiederholte Einwirkung des Spiritus brachte dasselbe an irgend einer Stelle, hier an der Gegend der Cardia, zum Einreissen, wozu die dabei ausgelösten Contractionen der Musculatur das ihrige beigetragen haben müssen. Der abgelöste Theil des Epithels hat nun als Fremdkörper eingewirkt, es kam zu Brechbewegungen. Die weitere Áblösung des schon gelockerten Epithels geschah auf die Weise, dass der zuerst abgelöste Theil durch die Brechbewegungen in das Lumen des Oesophagus eingestülpt und durch die immer weitere Abhebung höher liegender Partien des Epithels bis zum Munde heraus befördert wurde. Dort wo eine vollständige Lockerung des Epithels von seiner Unterlage nicht vorhergegangen war, kam es

1) Ueber die Intercellularlücken und Intercellularbrücken im Epithel. Zeitschrift für wissenschaft]. Zoologie. Bd. 41. S. 302, 
zu gewaltiger Abhebung desselben, daher das Bluterbrechen des Patienten und die constatirten kleinen Blutungen an der ausgestossenen Epithelröhre. Dass das Zustandekommen der Entleerung letzterer auf diese Weise erfolgte, geht aus dem mitgetheilten und noch mehr daraus hervor, dass die normalerweise dem Lumen des Oesophagus zugekehrte Fläche des Epithels an der ausgestossenen Röhre nach aussen zu liegen kam und dass bei der normalen Länge des Oesophagus von $25 \mathrm{~cm}$ es unmöglich wäre, über $10 \mathrm{~cm}$ der ausgestossenen Röhre zum Munde heraus zu hängen, wenn die Ablösung an der Cardia nicht begonnen hätte.

Aus dem Gesagten geht hervor, dass die Oesophagitis dissecans superficialis durch äussere, chemische Einwirkungen zu Stande kommen kann. Ferner dass die Zellen der Keimschicht des Epithels des Oesophagus des Menschen sich durch protoplasmatische Brücken verbinden, ebenso wie die Zellen des Stratum Malpighii der Haut, und dass die Eleidinkörner auch im Epithel des Oesophagus des Menschen vorkommen.

\section{Erklärung der Abbildungen. Tafel VI.}

Fig. 1. Die röhrenförmig ausgestossene Epitheldecke der Oesophagusschleimbaut. Die äussere Fläche derselben entspricht der normaler Weise dem Lumen des Oesophagus zugekehrten.

Fig. 2. Syst. VII. Oc. III. K. T. Leitz. Querscbnitt durch das Epithel der ausgestossenen Röhre. Intercellularlücken und -brücken. Vacuolen im Zellinnern.

Fig. 3. Syst. VII. Oc. III. K. T. Leitz. Querschnitt aus der obersten Epithelschicht. Eleidin- oder Keratohyalinkörner in den Epithelzellen.

Fig. 4. Subepitheliales Gewebe der unteren Fläche des Epithels der ausgestossenen Röhre anliegend. Die körnige Substanz, Leukocyten, Blutungen zu sehen. 